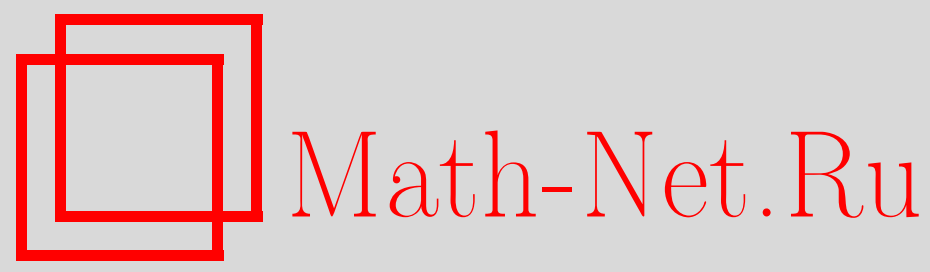

Р. К. Романовский, С. Г. Садовничук, Прямой метод построения двухзонных решений нелинейных уравнений, $M a-$ тем. заметки, 1999, том 66, выпуск 3, 401-406

DOI: https://doi.org/10.4213/mzm1181

Использование Общероссийского математического портала Math-Net.Ru подразумевает, что вы прочитали и согласны с пользовательским соглашением http://www . mathnet.ru/rus/agreement

Параметры загрузки:

IP : 54.157 .27 .8

26 апреля 2023 г., $15: 32: 22$

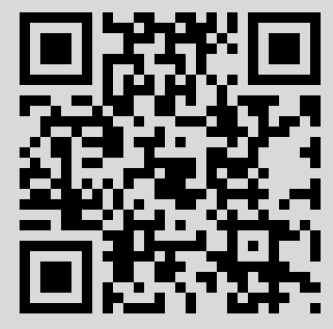




\title{
ПРЯМОЙ МЕТОД ПОСТРОЕНИЯ ДВУХЗОННЫХ РЕШЕНИЙ НЕЛИНЕЙНЫХ УРАВНЕНИЙ
}

\author{
Р. К. Романовский, С. Г. Садовничук
}

\begin{abstract}
Предлагается конструкция, позволяющая эффективно строить двухзонные решения, в том числе вещественные, некоторых нелинейньх уравнений без применения аппарата алгебраических кривых. Исходным элементом конструкции является специальная теорема сложения для тэта-функций двух переменных. Метод проиллюстрирован на уравнениях КП, Кд $\Phi$, sine-Gordon.

Библиография: 6 названий.
\end{abstract}

Введение. Построение решений нелинейных уравнений математической физики методами алгебраической геометрии предполагает последующую эффективизацию полученных формул [1]-[4]. В настоящей работе выполняется конструкция, позволяющая единообразным образом строить классы двухзонных решений, в том числе вещественных, некоторых нелинейных уравнений без применения аппарата алгебраических кривых. Отправным пунктом конструкции является специальная теорема сложения для тэта-функций двух переменных. Следствия из этой теоремы позволяют, в частности, эффективно вычислять векторы $U, V$ в тэта-функциональных формулах вида $u=f(U x+$ $V t)$ для решений. Строится треугольник в $\mathbb{C}^{2}$, сторонами которого являются с точностью до скалярных множителей градиенты при $z=0$ некоторых нечетных тэта-функций; искомые $U, V$ - надлежаще выбранные линейные комбинации нормалей к сторонам этого треугольника. Строящиеся решения становятся вещественными, если исходная матрица Римана связана с характеристиками участвующих в построениях тэта-функций специальными соотношениями. На этом пути построены классы решений уравнений sine-Gordon, КдФ, Кадомцева-Петвиашвили (КП).

Отметим, что изложенная в работе конструкция отличается от конструкции из [2], основанной на аппарате вешественных алгебраических кривых рода 2.

1. Две леммы. Пусть $g \in \mathbb{N}, B$ - матрица Римана порядка $g\left(B^{T}=B\right.$, $\operatorname{Im} B>0), I=\left\{\alpha \beta: \alpha, \beta \in\left(\mathbb{Z}_{2}\right)^{g}\right\} ; \theta_{\alpha \beta}(z)$-тэта-функция с характеристикой $(\alpha / 2, \beta / 2)$ и матрищей Римана $B$ :

$$
\theta_{\alpha \beta}(z)=\sum_{n \in \mathbb{Z}^{g}} \exp \{\pi i B(n+0.5 \alpha) \cdot(n+0.5 \alpha)+2 \pi i(n+0.5 \alpha) \cdot(z+0.5 \beta)\} .
$$

Здесь элементы $\mathbb{C}^{g}-$ столбцы; $a \cdot b=\sum a_{i} b_{i}$. Далее $e=(1, \ldots, 1)$ длины $g, \widehat{\alpha}=e-\alpha$, где $\alpha \in\left(\mathbb{Z}_{2}\right)^{g}$. Пару $\alpha \beta \in I$ будем также называть характеристикой. 
Лемма 1. При любьх $a b \in I, z, u \in \mathbb{C}^{g}$ верно равенство

$$
2^{g} \theta_{a \widehat{b}}(z+u) \theta_{a \widehat{b}}(z-u) \theta_{a b}^{2}(0)=\sum_{\alpha \beta \in I}(-1)^{b \cdot \alpha+a \cdot \beta} \theta_{\alpha \widehat{\beta}}^{2}(z) \theta_{\alpha \beta}^{2}(u) .
$$

ДокАЗАТЕЛЬСТво проводится вычислением по тэта-формуле Римана [5].

Для дальнейшего существенно, что слагаемые в правой части (2) с точностью до знака не зависят от выбора $a b$. Следующая лемма позволяет далее выделять вещественные решения.

Лемма 2. Пусть в (1) $\operatorname{Re} B=\Lambda$. Еслu

$$
2 \Lambda \in \operatorname{Mat}(g, \mathbb{Z}), \quad \Lambda \alpha+\operatorname{diag} \Lambda \in \mathbb{Z}^{g},
$$

$m o \overline{\theta_{\alpha \beta}(\bar{z})}=\xi(\alpha) \theta_{\alpha \beta}(z)$, əде $\xi(\alpha)=\exp \{-0.5 \pi i \Lambda \alpha \cdot \alpha\}$.

ДокАЗАТЕЛЬСТВо. Пусть $\Lambda=\left\|\lambda_{i j}\right\|$. Вьполняя при вычислении $\overline{\theta_{\alpha \beta}(\bar{z})}$ под знаком суммы замену $n \rightarrow-(n+\alpha)$ и принимая во внимание $\bar{B}=2 \Lambda-B$, получим $\overline{\theta_{\alpha \beta}(\bar{z})}=$ $\xi(\alpha) \sum_{n \in \mathbb{Z}^{g}} \exp \left(-2 \pi i a_{n}\right) \omega_{n}(z)$, где $a_{n}=(\Lambda \alpha+\operatorname{diag} \Lambda) \cdot n+2 \sum \lambda_{i j} n_{i} n_{j}, \omega_{n}(z)-n$-й член ряда (1). При условии (3) $a_{n}$ - целое число.

2. Специальная теорема сложения. Следствия. При $g=2$ множество $I$ состоит из 10 четных характеристик $a b$ ( $a \cdot b$ - четное число) и 6 нечетных. Перебором десяти вариантов просто устанавливается

ЛЕмма 3. При $g=2$ каждой четной $a b \in I$ отвечает единственное разбиение множества нечетных характеристик на два подмножества $\left\{\alpha_{k} \beta_{k}, k=1,2,3\right\}$ такое, что для каждого подмножества

$$
\alpha_{1} \beta_{1}+\alpha_{2} \beta_{2}+\alpha_{3} \beta_{3}=a b \bmod 2 \mathbb{Z}^{4}
$$

При $а b=\left(a^{\prime}, a^{\prime \prime} ; b^{\prime}, b^{\prime \prime}\right)$ одно из подмножеств дается формулами (сложение по $\bmod 2$ )

$$
\begin{gathered}
(1,1 ; 0,1),\left(1+a^{\prime} b^{\prime}, a^{\prime}\left(1+b^{\prime}\right)+a^{\prime \prime} ; 1, a^{\prime}\left(b^{\prime}+b^{\prime \prime}\right)+b^{\prime \prime}\right) \\
\left(a^{\prime}\left(1+b^{\prime}\right), 1+a^{\prime}\left(1+b^{\prime}\right) ; 1+b^{\prime}, 1+a^{\prime}\left(b^{\prime}+b^{\prime \prime}\right)\right) .
\end{gathered}
$$

Далее нумерация характеристик в каждом из двух нечетных наборов (4) предполагается произвольно фиксированной.

Tеорема 1. Пусть $g=2,\left\{\alpha_{k} \beta_{k}\right\}$ - любой из двух наборов (4), построенных по четной характеристике $a b \in I, \theta_{a b} \neq 0$. Тогда

$$
\begin{aligned}
\theta_{a \widehat{b}}(z+u) & \theta_{a \widehat{b}}(z-u) \\
& =\theta_{a b}^{-2}(0)\left(\theta_{a \widehat{b}}^{2}(z) \theta_{a b}^{2}(u)+\sum_{k=1}^{3}(-1)^{b \cdot \alpha+a \cdot \beta} \theta_{\alpha_{k} \widehat{\beta}_{k}}^{2}(z) \theta_{\alpha_{k} \beta_{k}}^{2}(u)\right) .
\end{aligned}
$$


ДокАЗАТЕльСтво. Пусть $\left\{a_{k} b_{k}\right\}$ - двойственный к $\left\{\alpha_{k} \beta_{k}\right\}$ набор (4). Складывая при $g=2$ равенство (2) для выбранной $a b \in I$ с такими же тремя равенствами для $a_{k} b_{k}$, умноженными соответственно на $(-1)^{b \cdot a_{k}+a \cdot b_{k}}$, с учетом нечетности $a_{k} b_{k}$ найдем

$$
\begin{gathered}
4 \theta_{a \widehat{b}}(z+u) \theta_{a \widehat{b}}(z-u) \theta_{a b}^{2}(0)=\sum_{\alpha \beta \in I} \varepsilon_{\alpha \beta} \theta_{\alpha \widehat{\beta}}^{2}(z) \theta_{\alpha \beta}^{2}(u), \\
\varepsilon_{\alpha \beta}=(-1)^{b \cdot \alpha+a \cdot \beta}+\sum_{k=1}^{3}(-1)^{b_{k} \cdot(a+\alpha)+a_{k} \cdot(b+\beta)} .
\end{gathered}
$$

Вычисления дают:

$$
\varepsilon_{\alpha \beta}= \begin{cases}4(-1)^{b \cdot \alpha+a \cdot \beta}, & \alpha \beta=a b, \alpha_{k} \beta_{k} ; \\ 0, & \alpha \beta \neq a b, \alpha_{k} \beta_{k} .\end{cases}
$$

Отсюда следует (5).

Введем матрицу $\wp_{a b}(z)=\left[\ln \theta_{a \widehat{b}}(z)\right]^{\prime \prime}$. Обозначим $\nabla_{k}=\theta_{\alpha_{k} \beta_{k}}^{\prime}(0), \sigma_{k}=(-1)^{b \cdot \alpha_{k}+a \cdot \beta_{k}}$, $\varphi_{k}(z)=\theta_{\alpha_{k} \widehat{\beta}_{k}}^{2}(z) \theta_{a \widehat{b}}^{-2}(z), k=1,2,3$.

СлеДСтвИЕ 1. В условиях теоремы 1 верно равенство

$$
\wp_{a b}(z)=\wp_{a b}\left(\frac{e}{2}\right)+\theta_{a b}^{-2}(0) \sum_{k=1}^{3} \sigma_{k} \nabla_{k} \nabla_{k}^{T} \varphi_{k}(z),
$$

где е $=(1,1), T$ - знак транспонирования $\left(\nabla_{k}-\right.$ столбииь $)$.

ДокАЗАТЕЛьСтво. Вычисляя матрицу Гесса по $u$ от обеих частей (5), подставляя $u=0$ и умножая полученное равенство на $0.5 \theta_{a \widehat{b}}^{-2}(z)$, получим: $\wp_{a b}(z)=A_{1}+A_{2}(z)$, где $A_{1}$ - постоянная матрица, $A_{2}(z)$ - второе слагаемое в правой части (6). Так как $\theta_{\alpha \widehat{\beta}}(e / 2)= \pm \theta_{\alpha \beta}(0)$ для нечетной $\alpha \beta$, то $A_{2}(e / 2)=0$ и $A_{1}=\wp(e / 2)$.

СледствиЕ 2. В условиях теоремы 1 для любого $\nu \in \mathbb{C}^{2}$

$$
D_{\nu}^{4} \ln \theta_{a \widehat{b}}(z)+6\left(D_{\nu}^{2} \ln \theta_{a \widehat{b}}(z)\right)^{2}=c_{\nu}+4 \theta_{a b}^{-2}(0) \sum_{k=1}^{3} c_{k \nu} \sigma_{k} \varphi_{k}(z)
$$

əде $D_{\nu}=\partial / \partial \nu, c_{k \nu}=\left(\nabla_{k} \cdot \nu\right) D_{\nu}^{3} \theta_{\alpha_{k} \beta_{k}}(0), \quad c_{\nu}=$ const.

ДокАЗАТЕЛЬСтво проводится вычислением $D_{\nu}^{4}$ от обеих частей $(5)$ как функций от $u$ и последующей подстановкой $u=0$ с учетом тождеств (см. [6])

$$
\begin{gathered}
D_{\nu}^{4}[f(u) f(-u)]_{u=0}=2\left[f D_{\nu}^{4} f-4 D_{\nu} f D_{\nu}^{3} f+3\left(D_{\nu}^{2} f\right)^{2}\right]_{u=0}, \\
f D_{\nu}^{4} f-4 D_{\nu} f D_{\nu}^{3} f+3\left(D_{\nu}^{2} f\right)^{2}=f^{2}\left[D_{\nu}^{4} \ln f+6\left(D_{\nu}^{2} \ln f\right)^{2}\right]
\end{gathered}
$$

и равенства $\left[D_{\nu}^{4} f^{2}-8 D_{\nu} f D_{\nu}^{3} f\right]_{u=0}=0$ для нечетной $f$.

Построенные по характеристикам (4) векторы $\nabla_{k}$ в общем положении образуют с точностью до скалярных множителей треугольник в $\mathbb{C}^{2}$ (попарно непараллельны). Построим нормали к сторонам этого треугольника по формулам

$$
v_{k}=\theta_{a b}(0)\left(\nabla_{i} \cdot J \nabla_{j}\right)^{-1} J \nabla_{k}, \quad k=1,2,3, \quad J=\left(\begin{array}{cc}
0 & 1 \\
-1 & 0
\end{array}\right),
$$

где $(i, j, k)$ - круговая перестановка чисел $1,2,3$. Очевидно, $v_{k} \cdot \nabla_{k}=0, \nabla_{i} \cdot J \nabla_{j}=$ $\operatorname{det}\left(\nabla_{i} \nabla_{j}\right) \neq 0$. Обозначим $D_{k}=\partial / \partial v_{k}$. 
Лемма 4. Верно равенство

$$
D_{i} D_{j} \ln \theta_{a \widehat{b}}(z)=\wp_{a b}\left(\frac{e}{2}\right) v_{i} \cdot v_{j}-\sigma_{k} \varphi_{k}(z) .
$$

ДокАЗАТЕЛЬСТво. Умножая обе части (6) справа на столбец $v_{i}$, затем скалярно на $v_{j}$ и принимая во внимание (8) и равенство $D_{i} D_{j} \ln \theta_{a \widehat{b}}=\wp_{a b} v_{i} \cdot v_{j}$, получим (9).

Нетрудно проверить: если в (4) $a \neq(0,0)$, то при некотором $k \in\{1,2,3\}$

$$
\alpha_{k}=a, \quad \alpha_{i}=\alpha_{j} \neq a .
$$

Из формул (8) и леммы 2 вытекает

ЛЕмма 5. Если характеристики (4) удовлетворяют условиям (10) и выполняются равенства (3) при $\alpha=a, \alpha_{i}$, то нормали $v_{i}, v_{j}, \xi(a) \xi^{-1}\left(\alpha_{i}\right) v_{k}$ вещественны.

ЗАмечАниЕ. При $\Lambda=0$ все нормали (8) вешественны. При $a \neq(0,0)$ для каждого из двух наборов (10) существует одна (по модулю целочисленного симметрического слагаемого) матрица $\Lambda \neq 0$, удовлетворяющая лемме 5. Для одного из наборов эта матрица удовлетворяет и условию $\xi\left(\alpha_{i}\right)= \pm \xi(a)$, в этом случае также все $v_{k} \in \mathbb{R}^{2}$.

\section{3. Построение решений уравнения sine-Gordon.}

TeOpema 2.

1. В условиях теоремы 1 функиия

$$
u(x, y)= \begin{cases}-\sqrt{-1} \ln \varphi_{k}\left(0.5 \sqrt{-1}\left(v_{i} x+v_{j} y\right)+z_{0}\right), & \sigma_{k}=-1, \\ -\sqrt{-1} \ln \varphi_{k}\left(0.5 \sqrt[4]{-1}\left(v_{i} x+v_{j} y\right)+z_{0}\right)+0.5 \pi, & \sigma_{k}=1,\end{cases}
$$

где $k \in\{1,2,3\},(i, j, k)$ - круговая перестановка из чисел $1,2,3, v_{i}, v_{j}$ - нормали (8), $z_{0} \in \mathbb{C}^{2},-$ решение уравнения

$$
u_{x y}=\sin u .
$$

2. Если выполнены условия леммы 5 и одновременно при этом $\operatorname{Re} z_{0}=0.25 \times$ $\left(\beta_{k}-b\right) \bmod 0.5 \mathbb{Z}^{2}$, то $\sigma_{k}=-1$ и первая функиия (11) - вещественное решение уравнения (12).

ДокАЗАТЕЛЬСТво. Подставляя в $(9) z_{1}=z+0.5\left(B\left(\alpha_{k}-a\right)+b-\beta_{k}\right)$ и используя свойство квазипериодичности тэта-функции [5, с. 102], после простых преобразований получим

$$
D_{i} D_{j} \ln \theta_{\alpha_{k} \widehat{\beta}_{k}}(z)=\wp_{a b}\left(\frac{e}{2}\right) v_{i} \cdot v_{j}+\varphi_{k}^{-1}(z) ;
$$

здесь $\varphi_{k}\left(z_{1}\right)=-\sigma_{k} \varphi_{k}^{-1}(z)$. Вычитая почленно (9) из (13), получим

$$
D_{i} D_{j} U=\left\{\begin{array}{ll}
4 \sin U, & \sigma_{k}=-1, r \\
-4 \sqrt{-1} \cos U, & \sigma_{k}=1,
\end{array} \quad U(z)=-\sqrt{-1} \ln \varphi_{k}(z) .\right.
$$

Отсюда следует, что обе функции (11) удовлетворяют (12). При условиях п. 2 теоремы $\sigma_{k}=(-1)^{a \cdot\left(b+\beta_{k}\right)}=-1, v_{i}, v_{j} \in \mathbb{R}^{2}$ и аргумент $z$ первой функции (11) удовлетворяет условию $\bar{z}=-z_{1}$. С учетом вытекающего из леммы 2 равенства $\overline{\varphi_{k}(z)}=\varphi_{k}(\bar{z})$ и четности $\varphi_{k}(z)$ имеем $\left|\varphi_{k}(z)\right|^{2}=\varphi_{k}(z) \varphi_{k}\left(z_{1}\right)=-\sigma_{k}=1$, откуда следует вещественность первой функции (11). 
4. Построение решений уравнения КдФ. Подставляя в (7) выражения для $\sigma_{k} \varphi_{k}(z)$, вычисленные из $(9)$, найдем

$$
\begin{cases}D_{\nu}^{4} \ln \theta_{a \widehat{b}}+6\left(D_{\nu}^{2} \ln \theta_{a \widehat{b}}\right)^{2}+4 L_{\nu}, & \theta_{a \widehat{b}}+\delta_{\nu}=0 \\ L_{\nu}=\sum_{(i, j, k)} \gamma_{k \nu} D_{i} D_{j}, & \gamma_{k \nu}=\theta_{a b}^{-2}(0)\left(\nabla_{k} \cdot \nu\right) D_{\nu}^{3} \ln \theta_{\alpha_{k} \beta_{k}}(0),\end{cases}
$$

где $(i, j, k)$ пробегает круговые перестановки чисел $1,2,3, \delta_{\nu}=$ const. Из формулы для $L_{\nu}$ следует:

$$
\nu \perp \nabla_{k} \Rightarrow L_{\nu}=D_{\nu} D_{\tau}, \quad \tau=\gamma_{i \nu} v_{j}+\gamma_{j \nu} v_{i}
$$

TEOPEMA 3.

1. При условии $\nu \perp \nabla_{k}$ функиия

$$
u(x, t)=\frac{\partial^{2}}{\partial x^{2}} \ln \theta_{a \widehat{b}}\left(0.5 \sqrt{2} \nu x+\sqrt{2} \tau t+z_{0}\right),
$$

әде $\tau$ - вектор $(15), z_{0} \in \mathbb{C}^{2},-$ решение уравнения

$$
u_{t}+6 u u_{x}+u_{x x x}=0 .
$$

2. Если выполнены условия леммы $5, \xi\left(\alpha_{i}\right)= \pm \xi(a), \nu=v_{k}, \quad z_{0} \in \mathbb{R}^{2}$, то решение (16) вешественно.

ДокАЗАТЕЛЬСТво. Обозначим $U(z)=D_{\nu}^{2} \ln \theta_{a \widehat{b}}(z)$. Применяя к $(14)$ оператор $D_{\nu}$, с учетом (15) получим

$$
D_{\nu}^{3} U+12 U D_{\nu} U+4 D_{\tau} U=0 .
$$

Далее, в условиях п. 2 теоремы в силу леммы 5 аргумент $z$ функции (16) вещественен и с учетом леммы $2 \overline{U(z)}=D_{\nu}^{2} \ln \left(\xi(a) \theta_{a \widehat{b}}(z)\right)=U(z)$. Из доказанного следуют оба утверждения теоремы.

5. Построение решений уравнения КП. Выберем $\nu, v \in \mathbb{C}^{2}$ так, что

$$
\nu \cdot \nabla_{k} \neq 0, \quad k=1,2,3, \quad \nu \nmid v .
$$

Разлагая нормали (8) по базису $\nu, v: v_{k}=s_{k} \nu+t_{k} v$, и подставляя в формулу для $L_{\nu}$ $D_{k}=s_{k} D_{\nu}+t_{k} D_{v}$, найдем

$$
L_{\nu}=D_{\nu} D_{\tau}+\psi D_{v}^{2}
$$

где

$$
\begin{aligned}
\tau & =\sum \gamma_{k \nu} s_{i} s_{j} \nu+\sum \gamma_{k \nu}\left(s_{i} t_{j}+s_{j} t_{i}\right) v \\
\psi & =(J \nu \cdot v)^{-2} \prod_{k=1}^{3}\left(\nu \cdot \nabla_{k}\right) \sum\left(J \nabla_{i} \cdot \nabla_{k}\right)^{-1}\left(\nabla_{j} \cdot J \nabla_{k}\right)^{-1} D_{\nu}^{3} \theta_{\alpha_{k} \beta_{k}}(0)
\end{aligned}
$$

суммирование ведется по круговьм перестановкам чисел $1,2,3$; в формуле для $\psi$ учтены значения $s_{k}, t_{k}$. При условиях $(17)$ в общем положении $\psi \neq 0$. Если, кроме того, $\nu, v \in$ $\mathbb{R}^{2}$, выполнены условия леммы 5 и при этом $\xi\left(\alpha_{i}\right)= \pm \xi(a)$ для характеристик (10), то $\tau \in \mathbb{R}^{2}, \psi \in \mathbb{R}$ и область $\left\{\nu, v \in \mathbb{R}^{2}: \psi>0\right\}$ непуста. В самом деле, в этом случае в силу леммы 5 все три нормали (8) вещественны, поэтому все $s_{k}, t_{k}$ вещественны. В силу леммы $2 \bar{\psi}=\psi$. Обозначим $\Delta_{k}=\left[\xi\left(\alpha_{k}\right)\right]^{0.5} \nabla_{k}, k=1,2,3$. Тогда $\bar{\Delta}_{k}=\Delta_{k}$, и $\psi$ представима в виде $\psi=\left(\nu \cdot \Delta_{1}\right)\left(\nu \cdot \Delta_{2}\right)\left(\nu \cdot \Delta_{3}\right) \rho(\nu, v)$, где все множители вешественны. Отсюда следует, что при фиксированном $v \psi(\nu)$ принимает в малых окрестностях $J \Delta_{k}$ значения любого знака. 
TEOPEMA 4.

1. При условиях (17) функиия

$$
u(x, y, t)=\frac{\partial^{2}}{\partial x^{2}} \ln \theta_{a \widehat{b}}\left(0.5 \sqrt{2} \nu x+\sqrt{2 \psi} v y+\sqrt{32} \tau t+z_{0}\right)+c
$$

әде $\tau, \psi$ даются формулами (19), $z_{0} \in \mathbb{C}^{2}, c \in \mathbb{C}-$ решение уравнения

$$
\left(u_{t}+6 u u_{x}+u_{x x x}\right)_{x}+u_{y y}=0 .
$$

2. Если выполнены условия леммы $5, \xi\left(\alpha_{i}\right)= \pm \xi(a)$ в $(10), z_{0} \in \mathbb{R}^{2}, c \in \mathbb{R} u$ векторы $\nu, v \in \mathbb{R}^{2}$ выбраны так, что $\psi>0$, то решение (20) вещественно.

ДокАЗАТЕЛЬСтво. Применяя к $(14) D_{\nu}^{2}$, с учетом $(18)$ получим

$$
D_{\nu}\left(D_{\nu}^{3} U+12 U D_{\nu} U+4 D_{\tau} U\right)+4 \psi D_{v}^{2} U=0
$$

где $U(z)$ та же, что в разделе 4 . Отсюда следует, что функция (20) - решение уравнения (21). В условиях п. 2 теоремы вещественность функции (20) проверяется также, как при доказательстве теоремы 3.

\section{СПИСОК ЦИТИРОВАННОЙ ЛИТЕРАТУРЫ}

[1] Дубровин Б. А. Тэта-функции и нелинейные уравнения // УМН. 1981. Т. 36. № 2. С. 11-80.

[2] Дубровин Б. А., Натанзон С. М. Вещественные двухзонные решения уравнения sine-Gordon // Функцион. анализ и его прилож. 1982. Т. 16. № 1. С. 27-43.

[3] Дубровин Б. А., Кричевер И. Н., Новиков С. П. Интегрируемыесистемы, 1 // Итоги науки и техники. Современные проблемы математики. Фундаментальные направления. Т. 4. М.: ВИНИТИ, 1985. С. 179-285.

[4] Бабич М.В. Эффективизация формул конечнозонного интегрирования уравнения sine-Gordon для одной кривой рода 3 // Функцион. анализ и его прилож. 1985. Т. 19. № 3. С. 53-55.

[5] Мамфорд Д. Лекции о тэта-функциях. М.: Мир, 1988.

[6] Хирота Р. Прямые методы в теории солитонов // Солитоны. М.: Мир, 1983. С. 175-192. 